Integrative taxonomy confirms two new West-Palaearctic species allied with Chrysotoxum vernale Loew, 1841 (Diptera: Syrphidae)

Zorica Nedeljković, Antonio Ricarte, Ljiljana Šašić Zorić, Mihajla Djan, Rüstem Hayat, Ante Vujić \& $\mathbf{M}^{a}$ Ángeles Marcos-García

Organisms Diversity \& Evolution

ISSN 1439-6092

Volume 20

Number 4

Org Divers Evol (2020) 20:821-833

DOI 10.1007/s13127-020-00465-w 
Your article is protected by copyright and all rights are held exclusively by Gesellschaft für Biologische Systematik. This e-offprint is for personal use only and shall not be selfarchived in electronic repositories. If you wish to self-archive your article, please use the accepted manuscript version for posting on your own website. You may further deposit the accepted manuscript version in any repository, provided it is only made publicly available 12 months after official publication or later and provided acknowledgement is given to the original source of publication and a link is inserted to the published article on Springer's website. The link must be accompanied by the following text: "The final publication is available at link.springer.com". 


\title{
Integrative taxonomy confirms two new West-Palaearctic species allied with Chrysotoxum vernale Loew, 1841 (Diptera: Syrphidae)
}

\author{
Zorica Nedeljković $^{1,2}$ (D) Antonio Ricarte ${ }^{2}$ - Ljiljana Šašić Zorić ${ }^{1} \cdot$ Mihajla Djan ${ }^{3} \cdot$ Rüstem Hayat $^{4} \cdot$ Ante Vujić $^{3} \cdot$ M $^{a}$ \\ Ángeles Marcos-García ${ }^{2}$
}

Received: 19 June 2019 / Accepted: 1 October 2020 / Published online: 3 November 2020

(C) Gesellschaft für Biologische Systematik 2020

\begin{abstract}
The taxonomy of the syrphid genus Chrysotoxum Meigen, 1803 (Diptera: Syrphidae), is complex and currently under scrutiny. Two new species allied with Chrysotoxum vernale, one from the Western Mediterranean, Chrysotoxum hispanicum sp. $\mathrm{n}$. and the other from the Eastern Mediterranean, Chysotoxum anatolicum sp. n., are described and illustrated. Chrysotoxum hispanicum sp. $\mathrm{n}$. is distinguished from the similar $C$. vernale Loew by the size of the yellow abdominal fasciae and shape of surstyli. Chrysotoxum anatolicum sp. n., known only from females, possesses an almost entirely yellow-pigmented wing, unusual amongst the other studied species of the $C$. vernale group. Additionally, C. hispanicum sp. n. and C. anatolicum sp. n. are separated from each other, as well as from other species of the $C$. vernale group by COI and ITS2 gene markers. An identification key to the West Palaearctic species of the C. vernale group is provided.
\end{abstract}

Keywords Adult morphology · COI · ITS2 · Chrysotoxum hispanicum sp.n. · Chrysotoxum anatolicum sp. n. · Identification key

\section{Introduction}

Within the family Syrphidae (Diptera), the subfamily Syrphinae (syrphines) consists of four tribes and 57 genera, 42 of which belong to the widespread tribe Syrphini (Mengual et al. 2008). Chrysotoxum Meigen, 1803, is one

Electronic supplementary material The online version of this article (https://doi.org/10.1007/s13127-020-00465-w) contains supplementary material, which is available to authorized users.

Zorica Nedeljković

zoricaned14@gmail.com

Antonio Ricarte

ricarte24@gmail.com

Ljiljana Šašić Zorić

ljsasic@biosense.rs

Mihajla Djan

mihajla.djan@dbe.uns.ac.rs

Rüstem Hayat

drhayat@gmail.com

Ante Vujić

ante.vujic@dbe.uns.ac.rs of the most distinctive Syrphini genera, with adults appearing as Batesian mimics of social wasps due to their long antennae, black and yellow bodies and oval to elongate, convex abdomens (Van Veen 2004). Larval biology is poorly known, but some species have been recorded in association with ant-attended root aphids (Aphididae)
Mángeles Marcos-García

marcos@ua.es

1 BioSense Institute-Research Institute for Information Technologies in Biosystems, University of Novi Sad, Dr Zorana Đinđića 1, Novi Sad 21000, Serbia

2 Centro Iberoamericano de la Biodiversidad (CIBIO), University of Alicante, 03690 San Vicente del Raspeig, Alicante, Spain

3 Faculty of Sciences, Department of Biology and Ecology, University of Novi Sad, Trg Dositeja Obradovića 2, Novi Sad 21000, Serbia

4 Faculty of Agriculture, Department of Plant Protection, Akdeniz University, 07059 Antalya, Turkey 
(Rotheray 1993). According to molecular phylogenetic work, Chrysotoxum is near Epistrophe Walker, 1852; Epistrophella Dušek et Láska, 1967; and Xanthogramma Schiner, 1861 (Mengual et al. 2008).

Chrysotoxum is widespread over all biogeographic regions except for Australasia and Antarctica, but is most speciose in the Palaearctic region with 85 described species (Thompson and Rotheray 1998; Mutin and Barkalov 1999; Huo and Zheng 2004; Huo and Ren 2007; Huo et al. 2006, 2007; Nedeljković et al. 2013, 2015, 2018a; Vujić et al. 2017). In the Western Palaearctic, i.e. excluding China and the Russian Far East (Lattin 1967; Van Steenis and Lucas 2011), there are 78 species, 13 of which are present in the Iberian Peninsula (Van Eck 2011; Ricarte and Marcos-García 2017) and 18 in Turkey, including the Anatolian Peninsula (Sarıbıyık 2014; Vujić et al. 2017; Nedeljković et al. 2018a). Iberian species of Chrysotoxum have not been revised yet, while those of the Balkan and Anatolian peninsulas have experienced scrutiny in recent years, with seven new species erected (Nedeljković et al. 2013, 2015, 2018a; Vujić et al. 2017).

Although identification of Chrysotoxum is usually straightforward at the genus level, separation of some species has proven difficult and often dependent on subtle morphological characters and molecular, morphometric or even ecological analyses (e.g. Nedeljković et al. 2013, 2015). In fact, some individual species have been split into more than one taxon (a species complex) as a product of an integrative study of their taxonomy. This is the case, for example, of Chrysotoxum vernale Loew, 1841, and the similar Chrysotoxum montanum Nedeljković et Vujić in Nedeljković et al. (2015) described from the Balkans.

The species $C$. vernale was regarded as part of the species group of Chrysotoxum festivum (Linnaeus, 1758) by Masetti et al. (2006). However, Nedeljković et al. (2015) and Vujić et al. (2017) started developing an implicit morphological concept of the C. vernale group for the species sharing a basoflagellomere shorter than scape and pedicel combined, pro- and mesofemora black basally and abdominal terga with yellow fasciae not reaching the lateral margins. Nonetheless, the bases of the pro- and mesofemora are sometimes dark yellow or with brown markings in $C$. orthostylum Vujić in Nedeljković et al. (2015).

Using both morphological and molecular characters, we examined Spanish and Turkish material of the C. vernale species group to determine its taxonomic status. Descriptions are provided for two new species, as well as a key to the Western Palaearctic species allied with $C$. vernale. The aim of this paper is to provide further steps in resolving the systematics of the genus Chrysotoxum by diagnosing and differentiating new taxa of the $C$. vernale group from the Western Palaearctic.

\section{Material and methods}

\section{Examined material}

A total of 52 specimens belonging to two potentially-new species were examined. These putative new species were compared with other species similar to $C$. vernale (Nedeljković et al. 2015; Vujić et al. 2017). The examined material is deposited in the following collections: Department of Biology and Ecology, Faculty of Sciences, University of Novi Sad (FSUNS); CIBIO Institute, University of Alicante, Spain (CEUA); Dieter Doczkal's private collection, Germany (DD). Acronyms of the repository collections are presented between square brackets (' $[$ ') after each series of specimens. Square brackets are also used when some additional information is provided, apart from that shown in the printed label. Abbreviations of the Spanish provinces, given between brackets in the examined material list, are as follows: CC, Cáceres; CR, Ciudad Real; GR, Granada; LE, León; O, Asturias; S, Santander; SA, Salamanca; V, Valencia. For location of the Spanish provinces, see map in Ricarte and Marcos-García (2017). In Cabañeros national park (Spain), specimens were collected with permits under the agreement to undertake research in the 040/2002 project ('Ministerio de Medio Ambiente', Spain). In Sierra Nevada national and natural parks collecting permits were issued by the 'Junta de Andalucía, Consejería de Medio Ambiente y Ordenación del Territorio' [Ref. number: ENSN/FJSG/IMJ (232)]. The fieldwork did not involve redlisted species, neither species protected in legislation.

\section{Morphological study}

To describe and diagnose species, characters were examined using a Nikon SMZ 745T and a Leica MZ12 binocular microscopes. Colour characters always refer to dry specimens. Body size was measured as the length ('L') from the tip of the frontal prominence (excluding antennae) to the tip of the abdomen. The proportional length of the antennal segments is given as a ratio (' $r$ ') of $\mathrm{x} / \mathrm{y} / \mathrm{z}$ (' $\mathrm{x}$ ', scape; ' $\mathrm{y}$ ', pedicel; ' $\mathrm{z}$ ', basoflagellomere). The length of each antennal segment was measured along its dorsal edge.

Male genitalia were removed from relaxed specimens and stored in microvials containing glycerol, after clearing in warm 10\% KOH for 3-5 min and then washing in acetic acid, followed by ethanol, to neutralize $\mathrm{KOH}$. Species were illustrated with photos produced as stacks of individual images made with a camera (Leica DFC 450) attached to a binocular stereomicroscope (Leica M205 C). Stacks and measurements were made with Leica Application Suite X (LAS X) ${ }^{\circledR}$, v. 3.0.4.16529. Morphological terms follow Thompson (1999), except for the term 'proepimeron', which follows Speight and Sarthou (2017). 'Tergum' is abbreviated as ' $T$ ' and 'sternum' as ' $\mathrm{S}$ ' in the species descriptions and diagnoses. 
New species were described based on the holotypes to warranty the stability of our species concepts by providing an unequivocal correlation between the descriptions and the name-bearing types (holotypes). The intraspecific variability found within the type series of each new species is recorded and included in different ways in the diagnoses, identification key and discussion.

\section{Molecular study}

In addition to the morphological character description, we performed molecular analyses using sequences from the 3 'COI region and the ITS2 region. We used the same matrix of concatenated COI and ITS2 sequences as in Nedeljković et al. (2018a) upgraded with the additional sequences of C. vernale group specimens. For detailed information on analysed specimens, see Table 1.

Protocols for DNA extraction, PCR amplification and sequencing of 3'COI and ITS2 regions are described in Nedeljković et al. (2018b). The newly-produced DNA sequences were edited for base-calling errors using BioEdit version 7.2.5. (Hall 1999). The rest of the sequences were downloaded from GenBank. COI sequences were aligned manually, whereas ITS2 sequences were aligned using the E-INS-I strategy as implemented in MAFFT v. 7 (Katoh and Standley 2013). Maximum-parsimony (MP) and maximumlikelihood (ML) phylogenetic trees were constructed for COI sequence matrix and for combined matrix of COI and ITS2 sequences. Parsimony analysis was performed in NONA (Goloboff 1999), spawned with the aid of ASADO (Nixon 2008) using the heuristic search algorithm with 1000 random addition replicates (mult_1000), holding 100 trees per round (hold/100), max trees set to 100,000 and applying tree-bisection-reconnection branch swapping. The ML tree was constructed using RAxML 8.2.8 (Stamatakis 2014) using the CIPRES Science Gateway web portal (Miller et al. 2010) under the general time-reversible (GTR) evolutionary model with a gamma distribution (GTRGAMMA) (Rodríguez et al. 1990). The bootstrap support values for clades were calculated with 1000 replicates for both MP and ML trees. The uncorrected pairwise distance (p) matrix for COI gene sequences and a number of variable positions were estimated using MEGA 7 software (Kumar et al. 2016). The trees were rooted on Melanostoma mellinum (Linnaeus, 1758) (GenBank accession numbers: KJ848101 for COI, KJ848059 for ITS2).

\section{Results}

\section{New species of the Chrysotoxum vernale group}

The two new species described here belong to the $C$. vernale species group as defined in the introduction.
Chrysotoxum anatolicum Nedeljković et Vujić, sp. $\mathbf{n}$. (Figs. 1a, 2, 4a, 5a)

Examined material. Holotype: 19, Turkey, İzmir, Bozdağ, Ski Centre 1, [38.332626 N 28.108250 E], 24 June 2016, leg. Vujić, Ačanski \& Uzal [FSUNS].

Paratype: 1 , , Turkey, İzmir, Bozdağ, Ski Center 1, [38.332626 N 28.108250 E], 25 June 2016, leg. Vujić, Ačanski \& Uzal [FSUNS].

Additional material of other species. Specimens of C. vernale and C. montanum, including types, published in Nedeljković et al. (2015).

Type locality. İzmir, Bozdağ (Turkey).

Diagnosis. $\mathrm{L}=14-15 \mathrm{~mm}(n=2)$. Gena dark brown to black; $r=1: 0.66: 1.42(n=2)$; basoflagellomere 3.28 times longer than wide $(n=2)$; pro- and mesofemora black in their basal thirds, metafemur yellow; wing extensively but unevenly pigmented, darker towards the anterior margin and apex: cells $R_{1}$ and $R_{2+3}$ dark yellow pigmented basally and dark brown to black pigmented apically, cells $\mathrm{R}_{4+5}$ and DM dark brown to black pigmented, cell $\mathrm{CuA}_{1}$ dark brown pigmented, cells $\mathrm{R}$ and $\mathrm{BM}$ dark yellow pigmented, cell $\mathrm{CuP}$ yellow pigmented (Fig. 4a).

Remarks. The other species of the $C$. vernale group, including C. hispanicum sp. n., have at least posterior half of wing without pigmentation. Chrysotoxum anatolicum sp. n. can also be separated from the similar $C$. vernale by the shape of the frontal pollinose maculae, bar-shaped in C. anatolicum sp. $\mathrm{n}$. (Fig. 1a) but triangular in C. vernale [Figure 5D in Vujić et al. (2017)], and colour of the posterior margin of T3, wholly black in $C$. anatolicum sp. n. but at least with a small central yellow macula in $C$. vernale.

Description. Female. $L=14 \mathrm{~mm}$.

Head (Figs. 1a, 2). Eye with sparse, straight, yellow pile; frons shiny black, with short, black pile and with two separate, bar-shaped, white pollinose maculae (Fig. 1a); ocellar triangle equilateral, shiny black with short black pile; occiput shiny black behind the ocellar triangle and white pollinose in other parts, narrowly yellow ventrally, with yellow pile intermixed with black pile dorsally; antenna black; scape and pedicel with black pile; $r=1: 0.62: 1.45$; arista bare, dark brown; face yellow, with yellow pile; face with a medial black vitta extending from antennal base to mouth edge; facial vitta as wide as a third of the facial width; face black postero-ventrally; gena yellow pilose, entirely shiny black (Fig. 2).

Thorax. Scutum black, with two medial, silvery, pollinose vittae extending for a third of the scutum length from its anterior margin, with short black pile; pleuron shiny black, except yellow maculae on posterior anepisternum and katatergum; katepisternum wholly black; scutellum yellow, except black on lateral corners and a medial macula; scutellum with short black pile; legs yellow except black on basal thirds of pro- and mesofemora; metafemur yellow; wing (Fig. 4a) 
Table 1 Chrysotoxum specimens genetically analysed, all deposited at FSUNS

\begin{tabular}{|c|c|c|c|c|c|c|c|}
\hline $\begin{array}{l}\text { DNA } \\
\text { ID }\end{array}$ & $\begin{array}{l}\text { 3'COI GenBank } \\
\text { accession number }\end{array}$ & $\begin{array}{l}\text { ITS2 GenBank } \\
\text { accession number }\end{array}$ & Species & Sex & Locality & Date & Leg. \\
\hline AU1254 & MH290053 & MH277392 & C. bozdagensis & $\hat{\sigma}$ & Turkey, Bozdağ, Ski Centre 1 & $25 / 06 / 2016$ & $\begin{array}{l}\text { Vujić A., Ačanski J. } \\
\text { \& Uzal A. }\end{array}$ \\
\hline AU1255 & MH290054 & MH277393 & C. bozdagensis & q & Turkey, Bozdağ, Ski Centre 1 & $24 / 06 / 2017$ & $\begin{array}{l}\text { Vujić A., Ačanski J. } \\
\text { \& Uzal A. }\end{array}$ \\
\hline $\mathrm{C} 27$ & MH290041 & KF408327 & C. festivum & 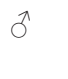 & $\begin{array}{l}\text { Serbia, Fruška Gora, above } \\
300 \mathrm{~m}\end{array}$ & $10 / 05 / 2011$ & Vujić A. \\
\hline $\mathrm{C} 19$ & MH290040 & KF408304 & C. festivum & q & $\begin{array}{l}\text { Serbia, Stara planina, Babin } \\
\text { zub }\end{array}$ & 09/07/2011 & Vujić A. \\
\hline $\mathrm{C} 29$ & MH290042 & KF408307 & C. festivum & 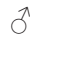 & $\begin{array}{l}\text { Serbia, Fruška Gora, above } \\
300 \mathrm{~m}\end{array}$ & $10 / 05 / 2011$ & Vujić A. \\
\hline $\mathrm{C} 35$ & MH290043 & KF408308 & C. festivum & q & $\begin{array}{l}\text { Serbia, Fruška Gora, above } \\
300 \mathrm{~m}\end{array}$ & $10 / 05 / 2011$ & Vujić A. \\
\hline $\mathrm{C} 40$ & MH290044 & KF408311 & C. elegans & $\hat{\sigma}$ & Serbia, Dubašnica, Demizlok & 08/06/2011 & Vujić A. \\
\hline $\mathrm{C} 43$ & MH290045 & KF408313 & C. elegans & $\widehat{\delta}$ & Serbia, Dubašnica, Demizlok & 08/06/2011 & Vujić A. \\
\hline $\mathrm{C} 44$ & MH290046 & KF408314 & C. elegans & q & $\begin{array}{l}\text { Montenegro, Prokletije, } \\
\text { Ropojanska dolina }\end{array}$ & Jul-2011 & Vujić A. \\
\hline $\mathrm{C} 47$ & MH290047 & KF408317 & C. elegans & q & $\begin{array}{l}\text { Serbia, Kopaonik, } \\
\quad \text { Samokovska reka }\end{array}$ & $02 / 08 / 2010$ & Vujić A. \\
\hline C51 & MH290048 & KF408320 & C. elegans & q & Serbia, Đerdap & 01/09/2011 & Vujić A. \\
\hline $\mathrm{C} 118$ & MH290051 & KF408339 & C. tomentosum & $\sigma^{\pi}$ & Serbia, Kopaonik, Kukavica & $14 / 07 / 2012$ & $\begin{array}{l}\text { Vujić A. \& } \\
\text { Radenković S. }\end{array}$ \\
\hline C119 & MH290052 & KF408340 & C. tomentosum & 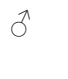 & $\begin{array}{l}\text { Serbia, Kopaonik, Marine } \\
\text { vode }\end{array}$ & $16 / 07 / 2012$ & $\begin{array}{l}\text { Vujić A. \& } \\
\text { Radenković S. }\end{array}$ \\
\hline $\mathrm{C} 22$ & MH290049 & KF408306 & C. tomentosum & $\widehat{\partial}$ & Serbia, Kopaonik, Metođe & 03/08/2010 & Vujić A. \\
\hline $\mathrm{C} 26$ & MH290050 & KF408326 & C. tomentosum & q & $\begin{array}{l}\text { Montenegro, Prokletije, } \\
\text { Ropojanska dolina }\end{array}$ & July-2011 & Vujić A. \\
\hline AU1252 & MW159863 & MW161162 & C. orthostylum & $\hat{0}$ & Turkey, Isparta & July-2015 & Vujić A. \\
\hline C104 & KR019026 & MH277388 & C. montanum & $\hat{o}$ & $\begin{array}{l}\text { Serbia, Kopaonik, Klisura } \\
\text { Samokovske reke }\end{array}$ & $16 / 06 / 2012$ & Vujić A. \\
\hline C105 & KR019027 & MH277389 & C. montanum & 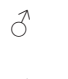 & $\begin{array}{l}\text { Serbia, Kopaonik, Klisura } \\
\text { Samokovske reke }\end{array}$ & $16 / 06 / 2012$ & Vujić A. \\
\hline $\mathrm{C} 13$ & KR019022 & MH277386 & C. vernale & 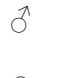 & $\begin{array}{l}\text { Greece, Olimp, Litochoras, } \\
\text { Prionia }\end{array}$ & $18 / 05 / 2011$ & Šimić S. \\
\hline $\mathrm{C} 3$ & KR019014 & MH277387 & C. vernale & 우 & Serbia, Malinik, Manastirište & $30 / 04 / 2011$ & Vujić A. \\
\hline $\mathrm{C} 12$ & KR019021 & MH277384 & $C$ vernale & q & $\begin{array}{l}\text { Greece, Olimp, Litochoras, } \\
\text { Prionia }\end{array}$ & $18 / 05 / 2011$ & Vujić A. \\
\hline $\mathrm{C} 130$ & KR019037 & MH277385 & C. vernale & $\hat{\sigma}$ & $\begin{array}{l}\text { Serbia, Đerdap, Ciganski } \\
\text { potok }\end{array}$ & $22 / 04 / 2012$ & Vujić A. \\
\hline MS155 & MW052731 & MW051571 & C. vernale & $0^{\lambda}$ & $\begin{array}{l}\text { Greece, Chelmos, Kalavryta } \\
\text { ski center }\end{array}$ & $06 / 06 / 2017$ & Vujić A. \\
\hline MS156 & MW052732 & MW051572 & C. vernale & $\sigma^{\pi}$ & $\begin{array}{l}\text { Greece, Chelmos, Kalavryta } \\
\text { ski center }\end{array}$ & 06/06/2017 & Vujić A. \\
\hline MS157 & MW052733 & MW051573 & C. vernale & $\hat{0}$ & $\begin{array}{l}\text { Greece, Chelmos, Kalavryta } \\
\text { ski center }\end{array}$ & 08/06/2017 & Vujić A. \\
\hline MS168 & MW052734 & MW051574 & $C$ vernale & q & Serbia, Vlasina, Delnice & $12 / 07 / 2017$ & Tot T. \& Vujić M. \\
\hline MS169 & MW052735 & MW051575 & C. vernale & 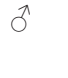 & Serbia, Zlot prema Maliniku & $24 / 07 / 2017$ & $\begin{array}{l}\text { Miličić M. \& } \\
\text { Ivošević B. }\end{array}$ \\
\hline MS170 & MW052726 & MW051566 & $\begin{array}{l}\text { C. hispanicum } \\
\text { sp. } \mathrm{n} \text {. }\end{array}$ & 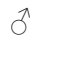 & $\begin{array}{l}\text { Spain, Sierra Nevada, first } \\
\text { valley }\end{array}$ & $17 / 06 / 2014$ & Vujić A. \\
\hline MS171 & MW052727 & MW051567 & $\begin{array}{l}\text { C. hispanicum } \\
\text { sp. } \mathrm{n} \text {. }\end{array}$ & q & $\begin{array}{l}\text { Spain, Ski Centar Sierra } \\
\text { Nevada }\end{array}$ & $16 / 06 / 2014$ & Vujić A. \\
\hline MS172 & MW052728 & MW051568 & $\begin{array}{l}\text { C. hispanicum } \\
\text { sp. } \mathrm{n} .\end{array}$ & 우 & $\begin{array}{l}\text { Spain, Ski Centar Sierra } \\
\text { Nevada }\end{array}$ & $16 / 06 / 2014$ & Vujić A. \\
\hline AU1256 & MW052729 & MW051569 & $\begin{array}{l}\text { C. anatolicum } \\
\text { sp. } \mathrm{n} .\end{array}$ & 우 & Turkey, Bozdağ, Ski Center 1 & $24 / 06 / 2016$ & $\begin{array}{l}\text { Vujić A., Ačanski J. } \\
\text { \& Uzal A. }\end{array}$ \\
\hline AU1257 & MW052733 & MW051570 & $\begin{array}{l}\text { C. anatolicum } \\
\text { sp. } \mathrm{n} \text {. }\end{array}$ & 우 & Turkey, Bozdağ, Ski Center 1 & $24 / 06 / 2016$ & $\begin{array}{l}\text { Vujić A., Ačanski J. } \\
\text { \& Uzal A. }\end{array}$ \\
\hline
\end{tabular}




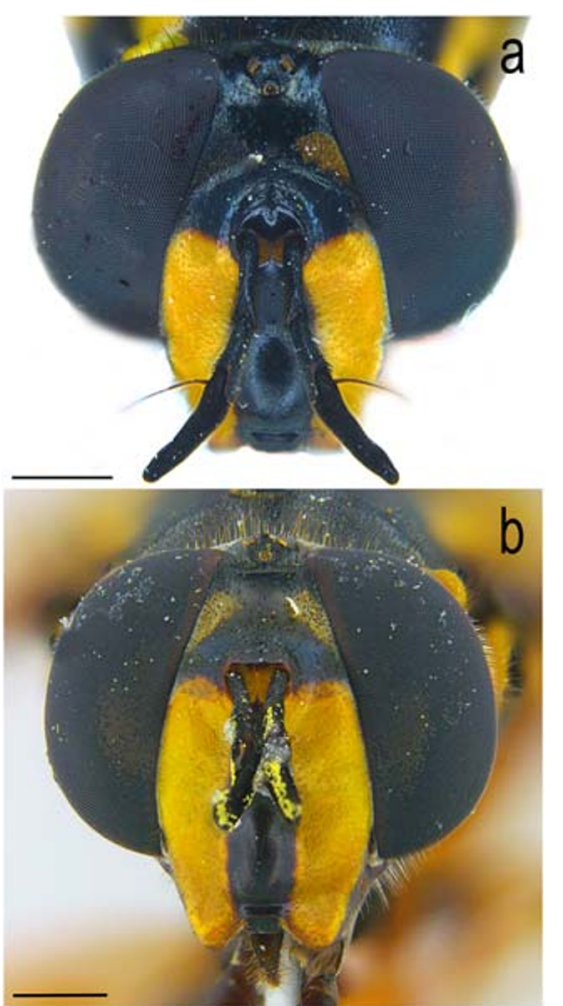

Fig. 1 Female heads of Chrysotoxum spp., frontal view. a C. anatolicum sp. n., İzmir, Turkey. b C. hispanicum sp. n., Salamanca, Spain. Scale lines: $0.75 \mathrm{~mm}$

membrane extensively microtrichose; wing cells $\mathrm{R}_{1}$ and $\mathrm{R}_{2+3}$ dark yellow pigmented basally and dark brown to black pigmented apically; cells $\mathrm{R}_{4+5}$ and $\mathrm{DM}$ dark brown to black pigmented; cell $\mathrm{CuA}_{1}$ dark brown pigmented; cells $\mathrm{R}$ and $\mathrm{BM}$ dark yellow pigmented; $\mathrm{CuP}$ yellow pigmented; calypter yellow with yellow pile at edge; halter dark brown basally, the remainder yellow, with lighter capitulum.

Abdomen (Fig. 5a). Shiny black, with short black pile, except for $\mathrm{T} 1$ and anterior part of $\mathrm{T} 2$ having long yellow and black pile intermixed; all terga with two separate, elongate, yellow maculae not reaching the lateral margins; yellow

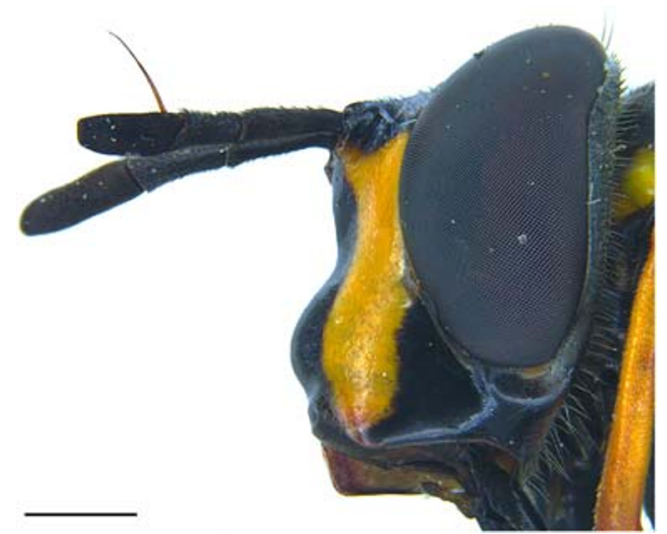

Fig. 2 Head of C. anatolicum sp. n., female, latero-ventral view. Scale line: $0.75 \mathrm{~mm}$.

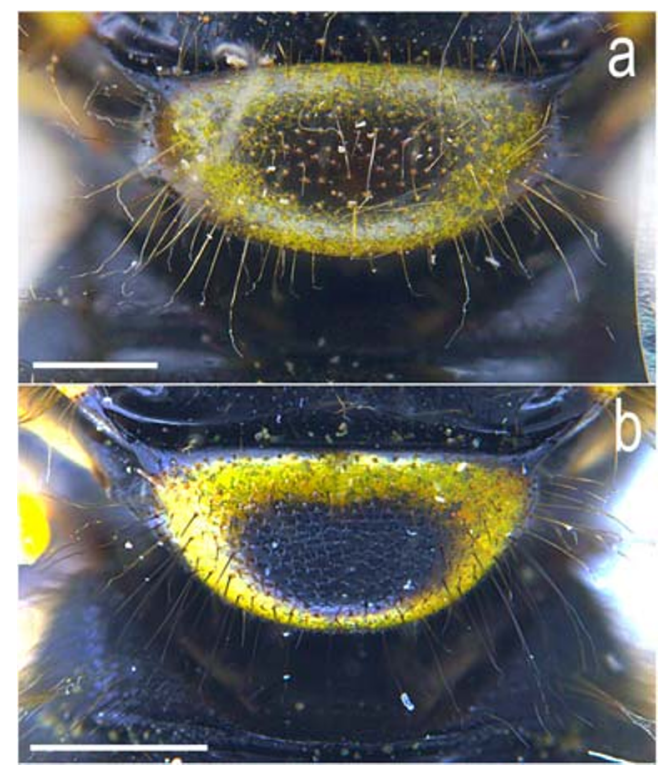

Fig. 3 Scutellums of Chrysotoxum spp., dorsal view. a C. hispanicum sp. n., holotype, male, Granada, Spain. b C. vernale, male, Dubašnica, Serbia. Scale lines: $0.5 \mathrm{~mm}(\mathbf{a}), 0.75 \mathrm{~mm}(\mathbf{b})$.

maculae bearing yellow pile; all sterna shiny black, with short yellow pile anteriorly and black pile posteriorly; S3 with two interconnected yellow maculae, not reaching the lateral margins; S4 with two yellow maculae not reaching the lateral margins; all sternal markings yellow pilose.

Male. Unknown.

Etymology. The specific epithet 'anatolicum' refers to the geographic origin of the specimens of the type series, a locality in the Anatolian Peninsula.

Habitats. Specimens were collected in a Mediterranean mountain steppe with Astragalus tmoleus var. tmoleus and Genista lydia var. lydia, between 1800 and $1900 \mathrm{~m}$ asl. This montane habitat is found at altitudes above forests of Pinus brutia and P. nigra pallasiana and below subalpinevegetation habitats (i.e. communities of Sideritis taurica, Euphorbia anacampseros var. tmolea, Minuartia juressi, Campanula teucrioides).

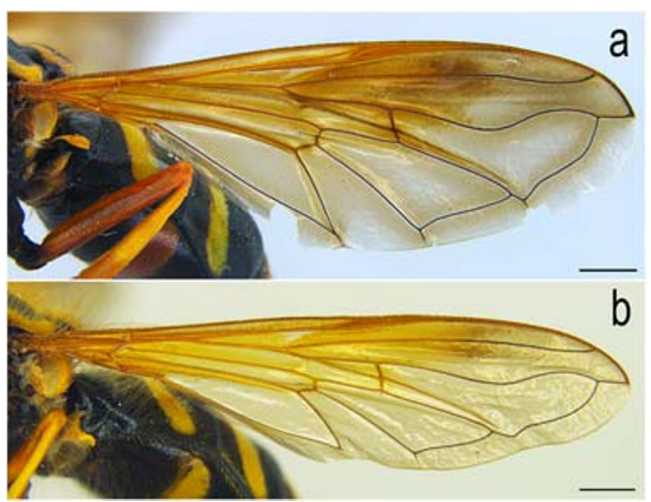

Fig. 4 Wings of Chrysotoxum spp. a C. anatolicum sp. n., female, İzmir, Turkey. b $C$. vernale, male (similar wing pigmentation as that found usually in C. hispanicum sp. n.), Bezdan, Serbia. Scale line: $1 \mathrm{~mm}$. 
Fig. 5 Abdomens of Chrysotoxum spp., dorsal view. a C. anatolicum sp. n., female, İzmir, Turkey. b C. hispanicum sp. n., holotype, male, Granada, Spain. c C. hispanicum sp. n., paratype, female, Salamanca, Spain. d C. vernale, male, Dubašnica, Serbia. Scale line: $1 \mathrm{~mm}$.
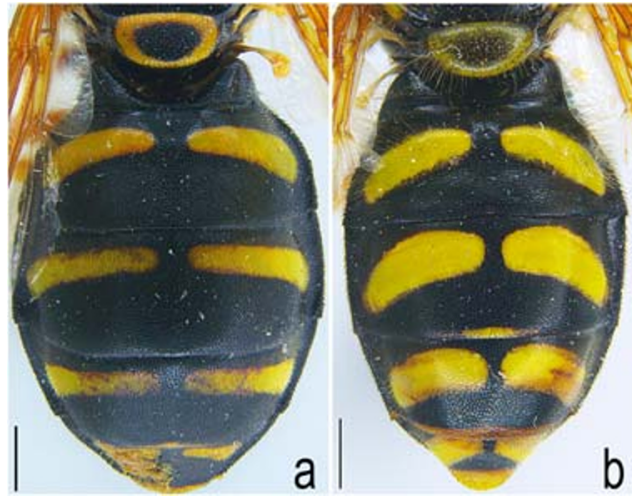

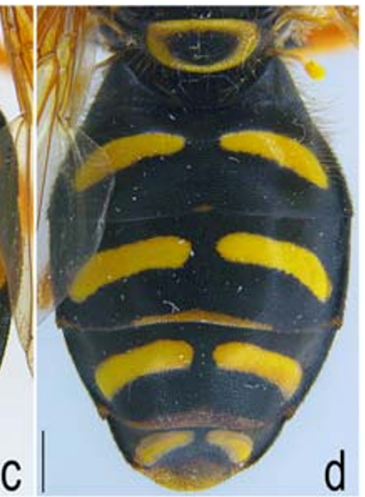

\section{Chrysotoxum hispanicum Nedeljković, Ricarte et Marcos-García, sp. n. (Figs. 1b, 3a, 5b-c, 6a-c)}

Examined material. Holotype: $1 \hat{\jmath}$, Spain, Sierra Nevada (GR), first valley ['Barranco de las Víboras', above 'Cabaña Sierra Nevada'], 17 June 2014 [3707'40”N 326'44”'W, $1626 \mathrm{~m}$ ] leg. Vujić [FSUNS].

Paratypes: $3 \delta^{\lambda} \partial^{\lambda}$, Andalucía, Sierra de Gador, Barjalí (AL), [2060 m], 10 June 2003 [3653'51”N 248'04”W], leg. D. Doczkal [S11374, S11375, S11376]; 4우, Andalucía, Sierra de Gador (AL), Barjalí, 2060m, 10 June 2003 [3653'51”N 2०48'04”W], leg. D. Doczkal [S11377, S11378, S11379, S11380] [DD]; 1, Sierra Nevada (GR), first valley ['Barranco de las Víboras', above 'Cabaña Sierra Nevada'], 17 June 2014, 3707'40”N 3²6'44”'W, 1626 m, leg. Vujić (07329); 2 우, Ski Centre, Sierra Nevada (GR),

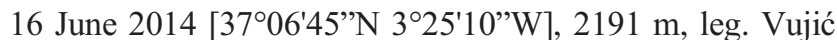
[MS171, MS172] [FSUNS]; 6ภํ, 2 우우, Chelva (V), 9-24

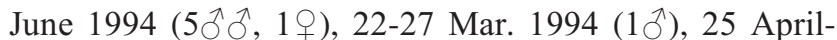
9 May 1994 (1q), leg. Pérez Bañón; 19, Utiel (V), 9 May 1994, leg. Pérez Bañón; 10̄, Requena (V), 7 Sept. 1994, leg. Pérez Bañón; 1q, Valverde del Fresno (CC), 950 m, 19 June 1981, leg. M.A. Marcos García; 1ð̄, Puerto El Torno (CC), 950m, 4 May 1982, leg. M.A. Marcos García; 1q, Hoyos (CC), 19 June 1981, leg. M.A. Marcos García; 19, La Garganta (CC), 1000m, 27 June 1980, leg. M.A. Marcos García; 19, P.N. de Cabañeros (CR), Pa1 [Valle de Santiago], 14 May 2004, leg. A. Ricarte; 1, P.N. de Cabañeros (CR), maJ1 [Melojar de Viñuelas], 14 April-8 May 2004, leg. A. Ricarte; 1ㅇ, La Alberca (SA), 17
Fig. 6 Male genitalia of Chrysotoxum spp., specimens from Spain. a C. hispanicum sp. n., epandrium, dorsal view (shape of each surstylus differs according to a slight view inclination). $\mathbf{b}$ C. hispanicum sp. n., hypandrium, lateral view. c C. hispanicum sp. n., right surstylus mounted in a slide. d C. vernale, right surstylus mounted in a slide. Scale lines: $250 \mu \mathrm{m}$ (a and b), $100 \mu \mathrm{m}$ (c and d).
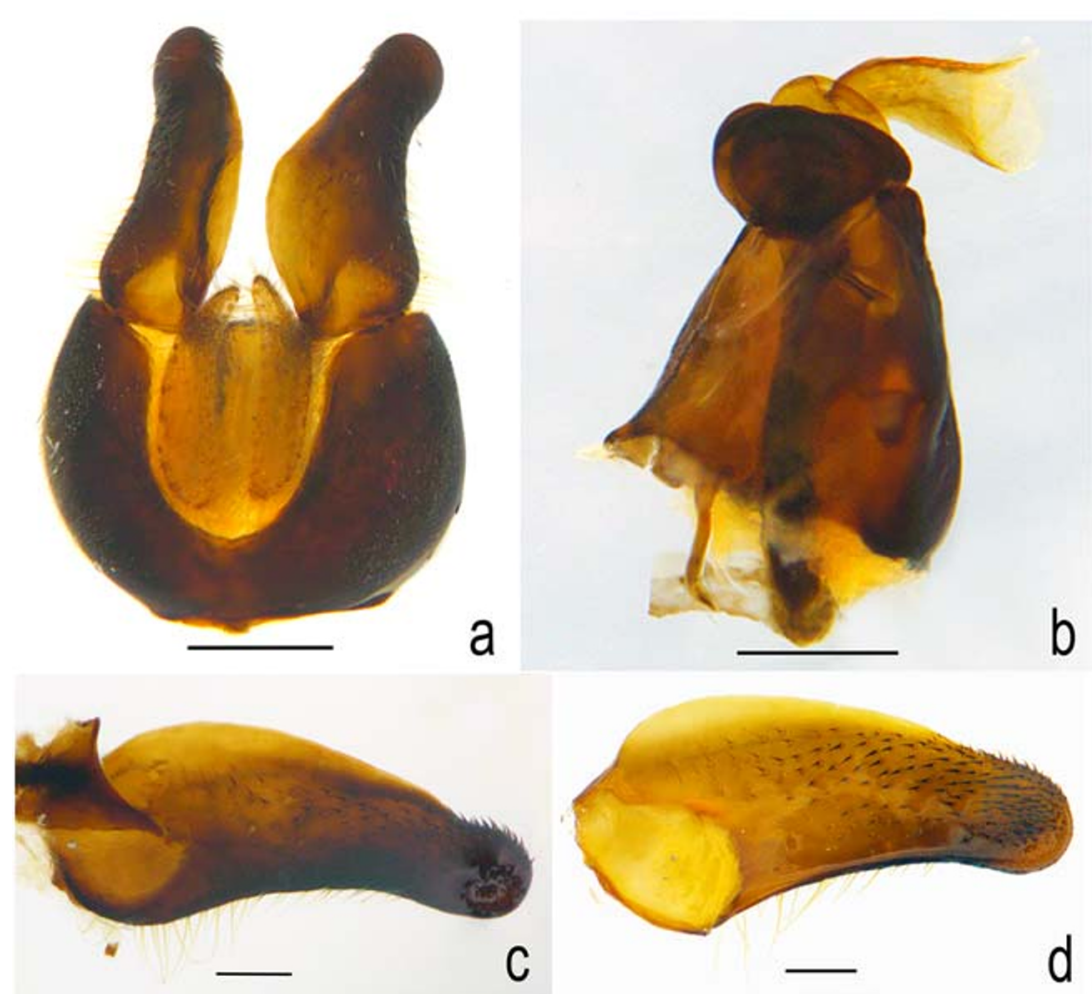
June 1980, leg. M.A. Marcos García; 19, Ledesma (SA), 26 June 1977, leg. M.A. Marcos García; $29 \circ$, Peñacaballera (SA), 18 May 1980, leg. M.A. Marcos García; 1ㅇ, Los Llanos, Bejar (SA), 30 May 1980, leg. M.A. Marcos García; 1 , , Monterrubio de la Armuña (SA), 11 June 1978, leg. M.A.

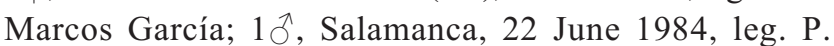
Calzada; $1 \circlearrowright^{\lambda}$, Navasfrías, Monte Mezas (SA), 19 June 1980, leg. M.A. Marcos García; 1 , , Negrilla de la Palencia (SA), 11 June 1978, leg. M.A. Marcos García; $2 \widehat{\partial}$ ô, Puerto Perales, El Payo (SA), 860 m, 24 May 1980, leg. M.A. Marcos García; 1, Escorial de la Sierra (SA), 15 May 1980, leg. M.A. Marcos García; 10ิ, Salamanca (SA), 7 May 1978, leg. M.A. Marcos García; 1ㅇ, Béjar (SA), 5 June 1977, leg. M.A. Marcos García; Puerto de Vallejera (SA), 26 June 1980, leg. M.A. Marcos García [CEUA]; 1 , Rinconada de la Sierra (SA), 17 June 1980, leg. M.A. Marcos García; 2 우, El Cabaco (SA), 29 June 1980, leg. M.A. Marcos García; 1 , Pino de Tormes (SA), 19 June 1979, leg. M.A. Marcos García; 1우 Puerto Magdalena (LE), 1434 m, 13 June 1986, leg. M.A. Marcos García; 1 웅 Pereda de Ancares (LE), 12 June 1986, leg. M.A. Marcos García; 1 , , Geras (LE), 3 June 1987, leg. M.A. Marcos García; 1ㅇ, Murias de Paredes (LE), 13 June 1986, leg. M.A. Marcos García; 1 + , Valdeteja (LE), 17 July 1977, leg. M.A. Marcos García.

Additional examined material of other species. Specimens of $C$. vernale and C. montanum, including types, published in Nedeljković et al. (2015). Spanish specimens morphologically fitting to the concept of $C$. vernale sensu Nedeljković et al. (2015): 1울, Plan d'Están, Valle Benasque (HU), 1850m, 26 July 1983, leg. M.A. Marcos García; 10̂, 1오, Plan de Sarrá, Valle de Benasque (HU), 1750m, 26 July 1983, leg. M.A. Marcos García; 1ð̄, 1, Sotres (S), 5 June 1988, leg. M.A. Marcos García; 1ð̂, Villar de Santiago (LE), 1 June 1988, leg. M.A. Marcos García; 1 9 , Tielve (O), 5 June 1988, leg. M.A. Marcos García; 1ㅇ, Hervás (CC), 17 May 1980, leg. M.A. Marcos García [CEUA].

Type locality. Sierra Nevada, Granada (Spain).

Diagnosis. $\mathrm{L}=9-12 \mathrm{~mm}(n=6)$. T2 yellow fascia about a third as wide as the width of the tergum; T3-T5 yellow fasciae about as half as wide as the width of the tergum (Figs. 5b-c); the long scutellum pile all yellow (Fig. 3a); gena at least partly yellow; $r=1: 0.85: 1.68(n=12)$; basoflagellomere 3.16 times longer than wide $(n=12)$.

Remarks. Abdomen with yellow maculae on terga much wider than those in C. montanum and C. vernale (Fig. 5d); the longest scutellum pile all yellow (sometimes just 1-2 long black pile present) (Fig. 3a), unlike C. vernale (Fig. 3b) and C. montanum which have all or most of the longest scutellum pile black; male genitalia in dorsal view, with surstylus apex more slender (Fig. 6c) than that in C. vernale (Fig. 6d).

Description. Male. $\mathrm{L}=9 \mathrm{~mm}$.
Head. Eye pile straight and yellow; vertical triangle black, with long, yellow pile (sometimes black pile also present anteriorly), white pollinose only in the posterior part of the triangle; frontal triangle about twice as long as region of eye contiguity, shiny black, with eye margins narrowly white pollinose from eye contiguity to antennae; frontal triangle with black pile anteriorly and mostly yellow pile posteriorly; antenna black, scape and pedicel with black pile, basoflagellomere 3.8 times longer than wide elongate and with rounded apex; arista bare and dark brown; $r=$ 1:0.66:1.43; face yellow pilose, yellow with a medial black vitta extending from the antennal bases to the mouth edge; facial black vitta as wide as a fourth of the facial width; mouth edge wholly but narrowly black; gena yellow, also with yellow pile; occiput white pollinose, with long yellow and black pile intermixed dorsally.

Thorax. Scutum black with two medial, silvery pollinose vittae extending from anterior margin for two thirds of the scutum length; scutum with intermixed long and short, yellow pile anteriorly, and with long, yellow and short, black pile posteriorly; pleuron shiny black except with yellow maculae on posterior anepisternum and katatergum; scutellum yellow, except for black lateral corners and a small medial macula, with long yellow pile (Fig. 3a); legs mainly yellow except for the black basal thirds of pro- and mesofemora; metafemora entirely yellow; wing membrane extensively microtrichose; wing cell $\mathrm{R}_{1}$ brown pigmented apically; cell $\mathrm{R}_{2+3}$ brown pigmented anteriorly; calypter yellow, with yellow pile at edge; halter yellow, with pedicel darker than capitulum.

Abdomen (Fig. 5b). Shiny black, with short black pile, except for the yellow pile on $\mathrm{T} 1$, anterior part of $\mathrm{T} 2$ and lateral parts of T3-T5; all terga with a yellow fascia interrupted in the middle; $\mathrm{T} 2$ yellow fascia as wide as a third of the tergum width; each T3-T5 yellow fasciae nearly as wide as half of the tergum width; yellow fasciae on terga with yellow pile; posterior margin of $\mathrm{T} 2$ black; posterior margin of $\mathrm{T} 3$ with a medial, narrow, yellow macula; posterior margin of T4 with a narrow, yellow fascia; T5 with a semicircular macula on the posterior margin; all sterna shiny black, $\mathrm{S} 1$ and anterior part of S2 with long yellow pile; posterior part of S2 and S3-S5 with adpressed black pile; S3 with two interconnected, yellow maculae not reaching the lateral margins; S4 with two yellow maculae not reaching the lateral margins. Genitalia as in Figs. 6a-c.

Female (Fig. 5c). Same as the male except for the frons having two separate triangular pollinose maculae on eye margins (Fig. 1b) and the scutum bearing short black pile.

Etymology. The specific epithet 'hispanicum' derives from Hispania, the Latin name for Spain, referring to the type locality of this species. Hispania was a Roman Empire province that also included the current Portugal. 
Distribution. Only confirmed from Spain, where it is apparently widespread (provinces: AL, CC, CR, GR, LE, SA, $\mathrm{V})$. It is found mainly in highlands, at altitudes from 500 to $2190 \mathrm{~m}$ asl.

Habitats. This species is found in a broad range of habitats, including mixed woodlands of Quercus faginea, $Q$. pyrenaica and $F$. angustifolia, and woodlands of $Q$. pyrenaica, both with temporary brooks and small grassy clearings (Cabañeros national park); 'dehesas' of $Q$. rotundifolia; croplands next to oak forests ( $Q$. rotundifolia). The holotype was collected in a meadow with a stream in the forests of 'Barranco de las Víboras', Sierra Nevada.

\section{Molecular analysis of the species allied with Chrysotoxum vernale}

We analysed $33 \mathrm{COI}$ gene sequences (including the outgroup) of $662 \mathrm{bp}$ in length. The ingroup sequences have 40 variable and 27 parsimony informative positions. The p-distances ratios range from 0.9 to $3 \%$, when comparing COI sequences between different species (Table S1). The topologies of COIbased ML and MP trees do not support the division between the $C$. festivum and the $C$. vernale species groups ( $C$. festivum group understood as a set of species with basoflagellomere shorter than scape and pedicel combined and pro- and mesofemora wholly yellow; $C$. vernale group sensu Introduction). Additionally, C. tomentosum Giglio-Tos, 1890, C. festivum and C. elegans Loew, 1841 are not resolved as separate. Chrysotoxum hispanicum sp. n. forms a nested clade with high bootstrap support (99/98) within the $C$. vernale clade, while the position of $C$. anatolicum $\mathrm{sp} . \mathrm{n}$. is questionable (Fig. 7a, b).

For the analyses on combined COI+ITS2 sequences, we produced a matrix of $1071 \mathrm{bp}$. The ingroup sequences have 73 variable positions from which 60 are parsimony informative. ML analysis based on COI+ITS2 resolved the $C$. vernale species group (C. vernale, C. montanum, C. orthostylum, C. hispanicum sp. n. and C. anatolicum sp. n.) as monophyletic with moderate bootstrap support (82) (Fig. 8a). The new species within $C$. vernale group, $C$. anatolicum sp. n. and C. hispanicum sp. n., are also resolved as monophyletic with medium to high clade bootstrap support (87 and 100). Chrysotoxum hispanicum sp. n. clade is nested within C. vernale clade but yet clearly divergent from it.

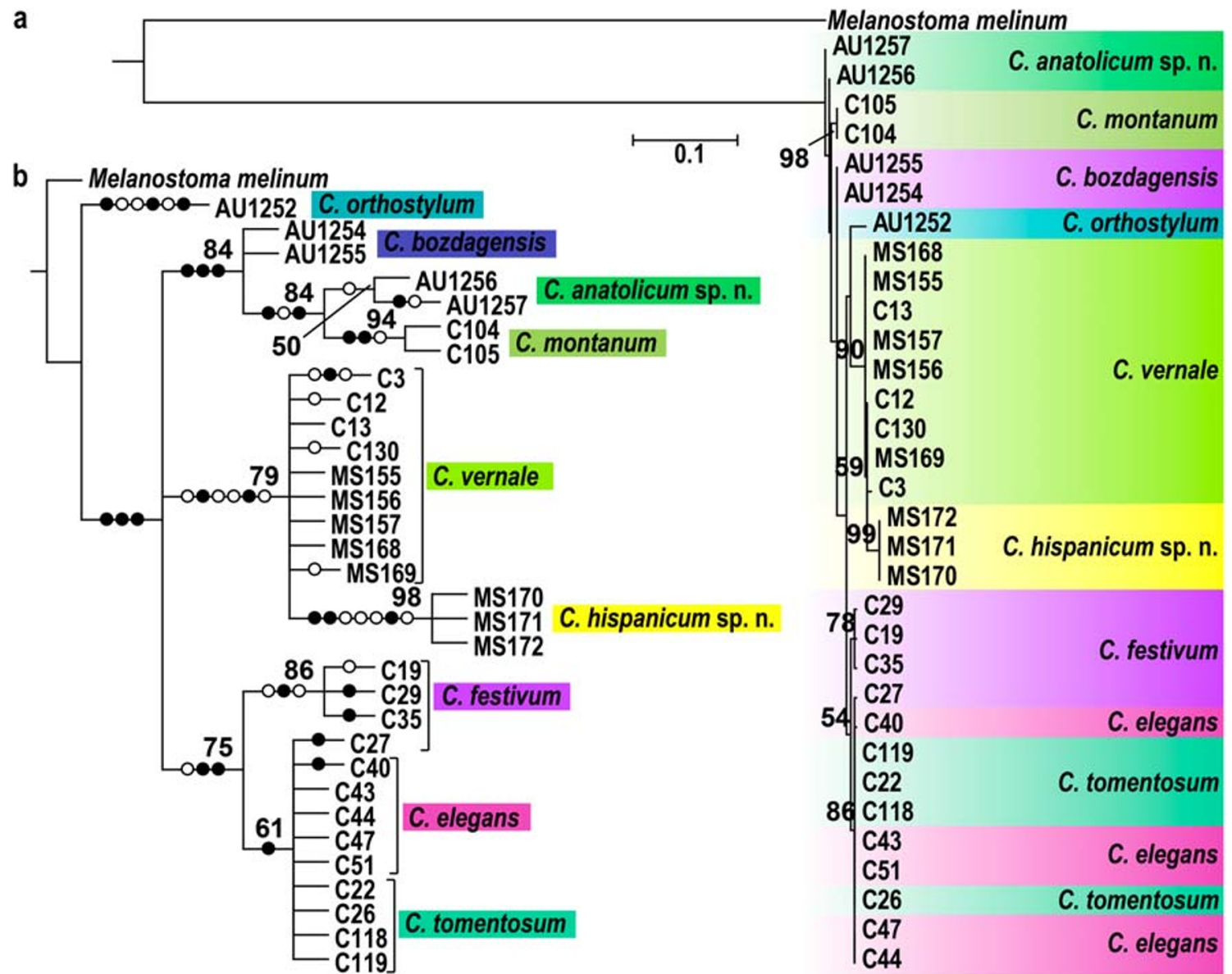

Fig. 7 COI gene trees of Chrysotoxum spp.. a Maximum likelihood tree. b Strict consensus of eight equally parsimonious trees.; filled circles denote non-homoplasious changes and open circles homoplasious changes. Length $=292$ steps; consistency index $=85$; retention index $=$ 90. Bootstrap values higher than 50 are indicated near nodes. 


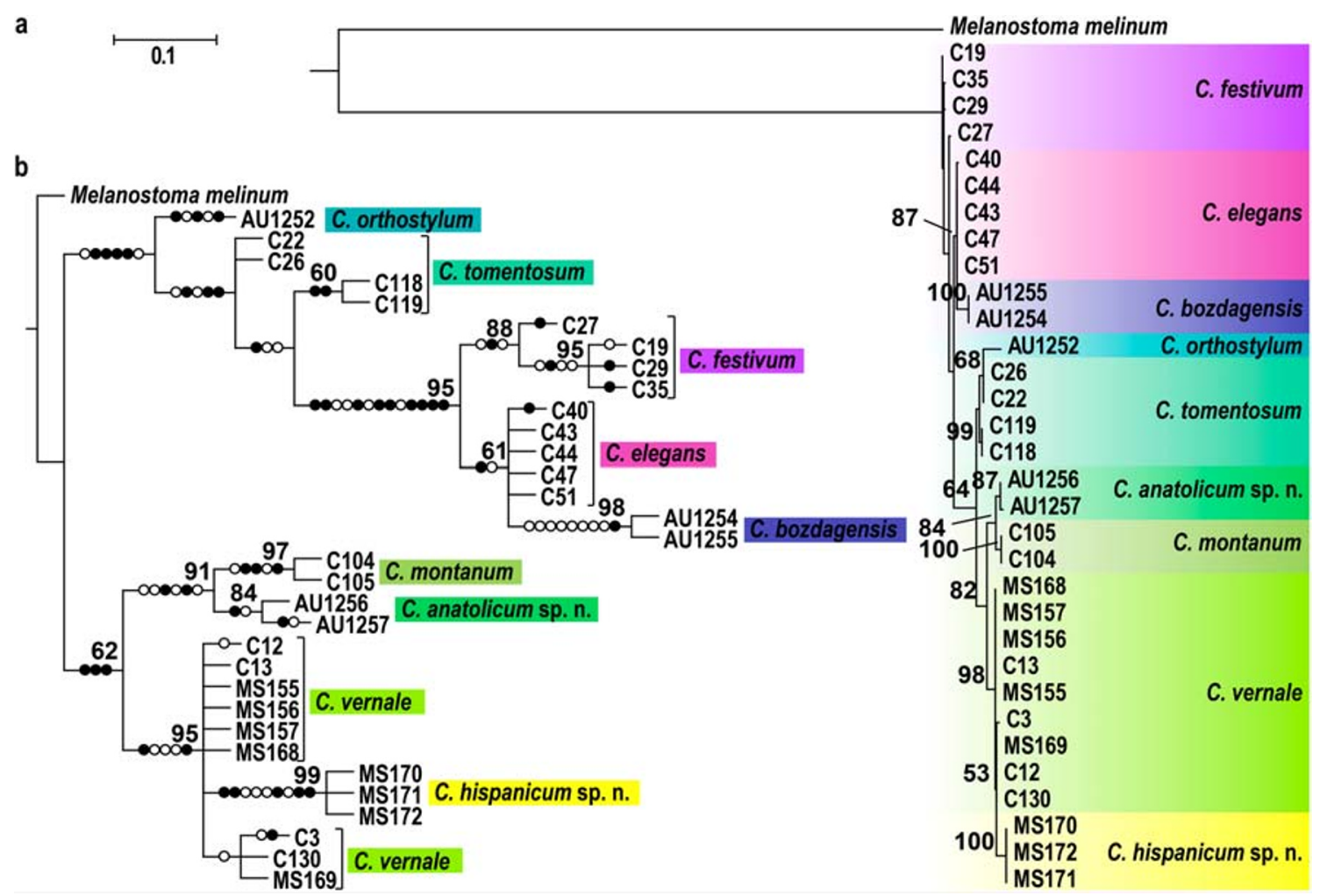

Fig. 8 Combined COI and ITS2 trees of Chrysotoxum spp.. a Maximum likelihood tree. b Strict consensus of four equally parsimonious trees; filled circles denote non-homoplasious changes and open circles homoplasious changes. Bootstrap values higher than 50 are indicated near nodes.

Maximum parsimony analysis based on COI+ITS2 produced four equally parsimonious trees of 279 steps in length (consistency index $=86$, retention index $=91$ ). The strict consensus tree shows two main clades (Fig. 8b). The first main clade comprises species of the $C$. festivum group [C. bozdagensis Nedeljković, Vujić et Hayat in Nedeljković et al. (2018a), C. tomentosum, C. festivum and C. elegans], but the bootstrap support for this clade is below 50 . The second main clade comprises the C. vernale group and has low bootstrap support (62). Chrysotoxum anatolicum sp. $\mathrm{n}$. and C. hispanicum sp. $\mathrm{n}$. are resolved as monophyletic with medium to high clade bootstrap support (84 and 99) as on ML tree, and C. hispanicum sp. n. clade is also nested within C. vernale clade.

\section{Key to the Western Palaearctic species of the Chrysotoxum vernale group}

The $C$. vernale group, understood as a set of species sharing a basoflagellomere shorter than scape and pedicel combined, pro- and mesofemora black basally and terga $2-4$ with yellow fasciae not reaching the lateral margins, consists now of the following eight species in the Western-Palaearctic: C. anatolicum sp. n., C. antennalis Vujić, Nedeljković et
Hayat in Vujić et al. (2017), C. clausseni Vujić, Nedeljković et Hayat in Vujić et al. (2017), C. hispanicum sp. n., C. lineare (Zetterstedt, 1819), C. montanum, C. orthostylum and $C$. vernale. All species of the C. festivum group (C. bozdagensis, C. elegans, C. festivum and C. tomentosum) (Figs. 7, 8), as well as light-legged specimens of $C$. orthostylum have pro- and mesofemora uniformly yellow or with their bases dark yellow. Light-legged specimens of $C$. orthostylum would key out with Nedeljković et al. (2015).

1a. Scape longer than pedicel; abdomen elongate .......... 2

1b. Scape shorter than pedicel; abdomen oval .................. 3

2a. Scutellum yellow, with a black central macula; mesofemora black in their basal thirds C. antennalis

2b. Black scutellum, with the posterior margin narrowly yellow; mesofemora black in their basal halves ... C. lineare

3a. Widest sections of the T3-4 yellow maculae extend for a third or more of each tergum length (Figs. $5 b-c)$

C. hispanicum sp. n.

3b. Widest sections of the T3-4 yellow maculae extend clearly less than a third of each tergum length (Figs. 5a, d) ... 4

4a. Posterior half of wing pigmented (Fig. 4a), including parts of cells $\mathrm{CuP}, \mathrm{CuA}_{1}$ and $\mathrm{DM}$ C. anatolicum sp. $\mathbf{n}$.

$4 \mathrm{~b}$. Posterior half of wing without pigmentation (Fig. 4b), at most pigmented in reduced areas of cells $\mathrm{CuP}, \mathrm{CuA}_{1}$ or DM ... 5 
5a. Anterior margin of scutum with two medial, silvery pollinose vittae extending for less than half of the scutum length; scutellum with a medial, transparent, dark-yellow macula C. clausseni

$5 b$. Anterior margin of scutum with two medial, silvery pollinose vittae extending for at least half of the scutum length, usually more; scutellum with a medial, opaque, black macula (Fig. 3b) .. 6

6a. Abdomen slender, T5 medial length more than half of the tergum maximum width; male surstylus gradually tapering towards the apex, more than 3 times longer than its maximum width C. orthostylum

6b. Abdomen stocky, T5 medial length half or less of the tergum maximum width (Fig. 5d); male surstylus expanded basally (Fig. 6d), less than 2.5 times longer than its maximum width 7

7a. Medial vittae of scutum evenly pollinose in their entire length; in male, long pile of scutum yellow; male surstylus less than 2.5 times longer than its maximum width (Fig. 6d); female with frontal pollinose maculae bar-shaped

C. vernale

7b. Medial vittae of scutum unevenly pollinose, less conspicuous in their posterior halves; in male, long pile of scutum black; male surstylus 3 times longer than its maximum width; female with frontal pollinose maculae triangular-shaped

C. montanum

\section{Discussion}

This study integrates information from both adult morphology and gene sequences to establish species limits within the Western Palaearctic species of the Chrysotoxum vernale group. The results confirmed the presence of two separate Chrysotoxum species at opposite sides of the Mediterannean Basin: C. hispanicum sp. n., from the Iberian Peninsula, and C. anatolicum sp. n., from the Anatolian Peninsula. Chrysotoxum hispanicum sp. $\mathrm{n}$. is distinguished from the similar $C$. vernale by the relative width of the yellow abdominal maculae, which are wider in relation to each tergum length in C. hispanicum sp. n. (Figs. 5b-c) than in C. vernale (Fig. 5d). The relative width of the yellow abdominal maculae proved to be useful to separate other Chrysotoxum species, for instance, C. festivum/C. tomentosum (Nedeljković et al. 2011, 2013) and C. bozdagensis/C. octomaculatum/C. elegans (Nedeljković et al. 2018a). The relative width of the abdominal maculae is also used in other syrphine genera such as Dasysyrphus Enderlein, 1938 and Epistrophe Walker, 1852 to separate species (Van Veen 2004; Doczkal and Schmid, 1994). Another useful character to separate $C$. hispanicum sp. n./C. vernale is the colour of the long scutellum pile, most of which are yellow in C. hispanicum sp. n. (Fig. 3a) and black in C. vernale (Fig. 3b).
Studied specimens of $C$. anatolicum sp. n. had a remarkable wing pigmentation pattern which covered most of the wing membrane (Fig. 4a), including cells $\mathrm{BM}, \mathrm{CuP}$ and $\mathrm{CuA}_{1}$. The other species of the $C$. vernale group usually lack pigmentation on the posterior part of wing (Fig. $4 b$ ): only a female of $C$. hispanicum sp. $\mathrm{n}$. was found to have pigmentation in the posterior part of wing but just in the membranous areas next to veins. Similar differences in the wing pigmentation are found in species of other syrphine genera such as Xanthogramma (Nedeljković et al. 2018b) and Leucozona Schiner, 1860 (Doczkal 1998, 2000). Another remarkable character in C. anatolicum sp. n. is the gena colour, which is wholly black, while in other species of the $C$. vernale group is at least partly pale. Nonetheless, some specimens of C. clausseni and C. montanum have a black gena.

Recent Chrysotoxum studies such as Nedeljković et al. (2018a) describing C. bozdagensis already showed the utility of a combined COI+ITS2 analysis for species delimitation in this genus. These two markers were also used independently, and with success, for resolving species within the $C$. festivum and $C$. vernale groups separately (Masetti et al. 2006; Nedeljković et al. 2013, 2015). However, in this study we showed a limited ability of COI gene sequences in resolving taxonomy of Chrysotoxum species when both $C$. vernale and C. festivum groups are analysed together. For example, C. festivum and C. tomentosum, both part of the C. festivum group, cannot be delimited using COI, but are resolved as separate using ITS2 sequences (Nedeljković et al. 2013). Thus, only analyses of combined COI and ITS2 sequences can provide successful delimitation of Chrysotoxum species. Unlike COI trees, $C$. vernale species group is resolved as monophyletic on combined COI+ITS2 trees, although with low to moderate bootstrap support $(\mathrm{ML}=82, \mathrm{MP}=62$ ). Additionally, C. hispanicum sp. n. and C. anatolicum sp. n. are resolved as genetically divergent from the other species of C. vernale group (Fig. 8). Chrysotoxum orthostylum does not belong to the $C$. vernale clade, neither to the $C$. festivum in the COI-based maximum parsimony tree (Fig. $7 b$ ), while in the tree based on COI+ITS2 groups together with species allied with $C$. festivum (Fig. 8b). In addition, the remarkable shape of the $C$. orthostylum male genitalia together with the reported variability in colour of pro- and mesofemora (Nedeljković et al. 2015) make the placement of this species difficult in any of the above mentioned species groups. Thus, we decide to leave $C$. orthostylum as an outlier within the genus Chrysotoxum until further systematic studies are undertaken.

Many syrphid species are rare and/or endemic to the Mediterranean Basin hotspot (e.g. Doczkal 1996; Vujić et al. 1999, 2001, 2007, 2011; Marcos-García et al. 2007, 2011; Ricarte et al. 2012; Grković et al. 2016), and the two new species described here further contribute to increase the biodiversity of this hotspot. Within the Mediterranean Basin, Turkey and mainland Spain have now a combined total of 
24 Chrysotoxum species, 19 in Turkey (Sarıbıyı 2014; Vujić et al. 2017; Nedeljković et al. 2018a; present study) and 14 in mainland Spain (Ricarte and Marcos-García, 2017; van Steenis et al. 2020; present study). The Spanish provinces of León and Salamanca have the highest numbers of Chrysotoxum species recorded in Spain (8 spp. each) (Ricarte and Marcos-García, 2017; present study), probably due to higher sampling efforts in these provinces. Italy and France equal mainland Spain in the number of Chrysotoxum species (14 spp.), but in countries further north, the numbers reduce to, for example, 11 in Germany, 8 in the UK and 7 in Norway, all three with well-studied hoverfly faunas (Speight et al. 2016). Turkey and mainland Spain share about 35\% (8 spp.) of their Chrysotoxum faunas, which is slightly higher than the percentage found in other syrphid genera such as Merodon Meigen (22\%) (Vujić et al. 2011). The number of Chrysotoxum species in the Iberian Peninsula will certainly increase after the $C$. vernale and other species groups such as that of $C$. intermedium Meigen, 1822 are revised (Nedeljković et al. in prep).

In Turkey, C. anatolicum sp. n. was found in Bozdağ, which extends as a mountain range (with the peak at $2157 \mathrm{~m}$ asl) for about $170 \mathrm{~km}$ in an east-west direction between the provinces of İzmir and Manisa (Toksöz and Ustaoğlu 2005). This locality includes forests with ancient chestnut (Castanea sativa), pines (Pinus brutia and Pinus nigra) and oaks (Quercus ithaburensis subsp. macrolepis and Quercus infectoria), Mediterranean scrublands ('macchia') and mountain steppes (Kurtaslan and Demirel 2011; Anlaş et al. 2010), all of them providing habitats and resources for a high diversity of invertebrates such as Coleoptera (Tenebrionidae) (Mercan et al. 2004), Dermaptera (Anlaş et al. 2010), Diptera (Chironomidae and Chaoboridae) and Oligochaeta (Toksöz and Ustaoğl 2005). At the moment, Bozdağ is the only known locality where both $C$. bozdagensis and C. anatolicum sp. n. occur (Nedeljković et al. 2018a).

Chrysotoxum hispanicum sp. $\mathrm{n}$. is widespread in the Iberian Peninsula and present in a broad variety of habitats. The records of $C$. vernale, confirmed here to occur in mainland Spain based on morphological evidence, originate from the Cantabrian Mountains (Provinces of Asturias, León and Santander) [Marcos-García (1990) in part], the 'Sistema Central' (Cáceres) [Marcos-García (1986) in part] and the Pyrenees (Huesca) (Marcos-García 1985), all high altitude mountain ranges. In Turkey, $C$. vernale is also found at high altitudes (2000-3000 m asl.), in the Erzurum Province (Sarıbıyık 2014; Vujić et al. 2017), while C. anatolicum sp. $\mathrm{n}$. is found in highlands but at a lower altitude $(1567 \mathrm{~m})$. Nonetheless, new studies may contribute to better define the altitudinal range where $C$. anatolicum $\mathrm{sp}$. n. occurs.

The detailed and often integrative study of the taxonomy of the $C$. vernale group in other parts of the Western Palaearctic (e.g. Balkan Peninsula) has also resulted in the definition and redefinition of several species concepts, some of them corresponding to new taxa (Nedeljković et al. 2015, Vujić et al. 2017). The present paper represents the first attempt to resolve the taxonomy of the $C$. vernale group in the Iberian Peninsula. As a result, $C$. hispanicum $\mathrm{sp}$. $\mathrm{n}$. has been discovered among material that was previously thought to belong to the species C. vernale (Marcos-García 1981, 1986, 1990; Ricarte and Marcos-García 2008). A higher level of taxonomic scrutiny of adults-i.e. further DNA, geometric morphometric, and ecological analyses in a more extensive study area-of Chrysotoxum populations, as well as a more complete knowledge of their early stages and biology are expected to increase the alpha diversity of this genus in the Iberian Peninsula and other parts of Western Europe (Speight 2018).

Funding This study was funded by the Ministry of Education, Science and Technological Development of the Republic of Serbia (Research Project No: OI173002), the H2020 project "ANTARES" (No: 664387), and the "Vicerrectorado de Investigación y Transferencia de Conocimiento' of the University of Alicante (Ref. UATAL05).

Data availability statement The datasets generated during and/or analysed during the current study are available from the corresponding author on reasonable request.

\section{Compliance with ethical standards}

Conflict of interest The authors declare that they have no conflict of interests.

Ethical approval All applicable international, national and international guidelines for the care and use of animals were followed.

This article does not contain any studies with human participants performed by any of the authors.

\section{References}

Anlaş, S., Haas, F., \& Tezcan, S. (2010). Dermaptera (Insecta) fauna of Bozdağlar Mountain, Western Turkey. Linzer Biologische Beiträge, 42(1), 389-399.

Doczkal, D. (1996). Description of two new species of the genus Eumerus (Diptera, Syrphidae) from Corsica. Volucella, 2, 3-19.

Doczkal, D. (1998). Leucozona lucorum (Linnaeus)- a species complex? (Diptera, Syrphidae). Volucella, 3(1/2), 27-49.

Doczkal, D. (2000). Redescription of Leucozona nigripila Mik and description of Leucozona inopinata spec. nov. (Diptera, Syrphidae). Volucella, 5, 115-127.

Doczkal, D., \& Schmid, U. (1994). Drei neue Arten der Gattung Epistrophe (Diptera: Syrphidae), mit einem Bestimmungsschlüssel für die deutschen Arten. Stuttgarter Beiträge zur Naturkunde, 507, $1-32$.

Dušek, J., \& Láska, P. (1967). Versuch zum Aufbau eines natürlichen Systems mitteleuropäischer Arten der Unterfamilie Syrphinae (Diptera). Acta scientiarum naturalium Academiae Scientiarum Bohemicae, Brno, 1, 349-390.

Enderlein, G. (1938). Beiträge zur Kenntnis der Syrphiden. Sitzungsberichte der Gesellschaft Naturforschender Freunde Berlin, 1937, 192-237. 
Grković, A., Vujić, A., Radenković, S., Chroni, A., \& Petanidou, T. (2016). Diversity of the genus Eumerus Meigen (Diptera, Syrphidae) on the eastern Mediterranean islands with description of three new species. Annales de la Société entomologique de France, 51, 361-373. https://doi.org/10.1080/00379271.2016. 1144483.

Giglio-Tos, E. (1890). Le specie europee del genere Chrysotoxum Meig. Atti della Reale Accademia delle scienze di Torino, 26, 134-165.

Goloboff, P. A. (1999). NONA computer program. Version 2.0. Tucuman (Argentina), www.softpedia.com/get/Scienece-CAD/ NONA.shtml/. Accessed 22 April 2018.

Hall, T. A. (1999). BioEdit: a user-friendly biological sequence alignment editor and analysis program for Windows 95/98/ NT. Nucleic Acids Symposia, 41, 95-98.

Huo, K. K., \& Ren, G. D. (2007). Investigation on syrphids (Diptera: Syrphidae) of Xiaowutai Shan Nature Reserve. Hebei Prov. with descriptions of new species and new recorded species. Entomotaxonomia, 20, 172-198.

Huo, K. K., Ren, G., \& Zheng, Z. (2007). Fauna of Syrphidae from Mt. Qinling-Bash in China (Insecta: Diptera). Beijing, Beijing Huonzheng Printer LTD Co.

Huo, K. K., Zhang, H. J., \& Zheng, Z. M. (2006). Descriptions of a new species and a new record species of Chrysotoxum (Diptera, Syrphidae) from China. Acta Zootaxonomica. Sinica, 31, 438-444.

Huo, K. K., \& Zheng, Z. M. (2004). Three new species of the genus Chrysotoxum Meigen from Shaanxi Province (Diptera, Syrphidae, Chrysotoxini). Acta Zootaxonomica Sinica, 29(1), 166-171.

Katoh, K., \& Standley, D. M. (2013). MAFFT Multiple Sequence Alignment Software Version 7: Improvements in performance and usability. Molecular Biology and Evolution, 30, 772-780.

Kumar, S., Stecher, G., \& Tamura, K. (2016). MEGA7: Molecular Evolutionary Genetics Analysis Version 7.0 for Bigger Datasets. Molecular Biology and Evolution, 33(7), 1870-1874.

Kurtaslan, B. O., \& Demirel, Ö. (2011). Pollution caused by peoples' use for socio-economic purposes (agricultural, recreation and tourism) in the Gölcük Plain Settlement at Bozdağ Plateau (Ödemiș-İzmir/ Turkey): a case study. Environmental Monitoring and Assessment, $175,419-430$.

Lattin G. de (1967). Grundriss der Zoogeographie. Jena, Germany: Gustav Fischer Verlag.

Linnaeus, C. (1758). Systema naturae per regna tria naturae, secundum classes, ordines, genera, species, cum caracteribus, differentiis, synonimis, locis. Ed. 10, Vol. 1. Stockholm: L. Salvii, Holmiae.

Loew, H. (1841). Ueber die Gattung Chrysotoxum. Stettiner Entomologische Zeitung, 2(136-141), 155-160.

Marcos-García, M. A. (1981). Contribución al conocimiento de los Syrphidae (Dipt.) de la zona noreste de la provincia de Salamanca. Boletín de la Asociación Española de Entomología, 4, 157-171.

Marcos-García, M. A. (1985). Contribución al conocimiento de la sirfidofauna del Pirineo del Alto Aragón I (Diptera, Syrphidae). Boletim da Sociedade Portuguesa de Entomologia, 1, 511-520.

Marcos-García, M. A. (1986). Los Syrphidae (Diptera) de las sierras occidentales del Sistema Central español: Subfamilias: Chrysotoxinae, Sphegininae, Chilosiinae, Pelecocerinae, Volucellinae y Cinxiinae. Boletín de la Asociación Española de Entomología, 10, 159-180.

Marcos-García, M. A. (1990). Catálogo preliminar de los Syrphidae (Diptera) de la Cordillera Cantábrica (España). Eos, 66, 81-235.

Marcos-García, M. A., Vujić, A., \& Mengual, X. (2007). Revision of Iberian species of the genus Merodon (Diptera: Syrphidae). European Journal of Entomology, 104, 531-572. https://oi.org/ 10.14411/eje.2007.073.

Marcos-García, M. A., Vujić, A., Ricarte, A., \& Ståhls, G. (2011). Towards an integrated taxonomy of the Merodon equestris complex (Diptera: Syrphidae) including description of a new species, with additional data on Iberian Merodon. The Canadian Entomologist, 143, 332-348. https://doi.org/10.4039/n11-013.

Masetti, A., Lucetti, A., Sommaggio, D., Burgio, D., \& Mantovani, B. (2006). Phylogeny of Chrysotoxum species (Diptera: Syrphidae) inferred from morphological and molecular characters. European Journal of Entomology, 103, 439-467.

Meigen, J.W. (1803). Versuch einer neuen Gattungseintheilung der europäischen zweiflügeligen Insekten. Magazin für Insektenkunde, 2, 259-281.

Meigen, J.W. (1822). Systematische Beschreibung der bekannten europäischen zweiflügeligen Insekten. Dritter Theil. SchulzWundermann, Hamm.

Mengual, X., Ståhls, G., \& Rojo, S. (2008). First phylogeny of predatory flower flies (Diptera, Syrphidae, Syrphinae) using mitochondrial COI and nuclear 28S rRNA genes: conflict and congruence with the current tribal classification. Cladistics, 24, 543-562. https:// doi.org/10.1111/j.1096-0031.2008.00200.x.

Mercan, T., Keskin, B., \& Tezcan, S. (2004). Bozdağ (Ödemiş, İzmir)' in Tenebrionidae (Coleoptera) Faunasının Çukur Tuzaklarla Belirlenmesi Üzerinde Bir Araștırma [An Investigation on the Determination of Tenebrionidae Fauna of Bozdağ (Ödemiş, İzmir) by Use of the Pitfall Traps]. Ekoloji, 14(53), 44-48.

Miller, M. A., Pfeiffer, W., Schwartz, T. (2010). Creating the CIPRES Science Gateway for inference of large phylogenetic trees. Proceedings of the Gateway Computing Environments Workshop (GCE), New Orleans, LA., 1-8.

Mutin, V. A., \& Barkalov, A. V. (1999). 62. Fam. Syrphidae: In P. A. Lehr (Ed.), Key to the insects of Russian Far East. Vol. 6. Diptera and Siphonaptera. Part 1. (pp. 342-500). Vladivostok, Dal'nauka.

Nedeljković, Z., Ačanski, J., \& Vujić, A. (2011). Chrysotoxum festivum (L., 1758) (Diptera: Syrphidae): a species complex? $6^{\text {th }}$ International Symposium on the Syrphidae, Abstract book, contribution 18.

Nedeljković, Z., Ačanski, J., Vujić, A., Obreht, D., Đan, M., Ståhls, G., \& Radenković, S. (2013). Taxonomy of Chrysotoxum festivum Linnaeus, 1758 (Diptera: Syrphidae) - an integrative approach. Zoological Journal of the Linnean Society, 169, 84-102. https:// doi.org/10.1111/zoj.12052.

Nedeljković, Z., Ačanski, J., Đan, M., Obreht-Vidaković, D., Ricarte, A., \& Vujić, A. (2015). An integrated approach to delimiting species borders in the genus Chrysotoxum Meigen, 1803 (Diptera: Syrphidae), with description of two new species. Contributions to Zoology, 84, 285-304. https://doi.org/10.1163/1875986608404002 .

Nedeljković, Z., Vujić, A., Hayat, R., Šašić Zorić, Lj., \& Đan, M. (2018a). A new species of the genus Chrysotoxum Meigen, 1803 (Diptera: Syrphidae) from Turkey. Zoology in the Middle East, 64, 244-252. https://doi.org/10.1080/09397140.2018.1475122.

Nedeljković, Z., Ricarte, A., Šašić Zorić, Lj., Đan, M., Obreht-Vidaković, D., \& Vujić, A. (2018b). The genus Xanthogramma Schiner, 1861 (Diptera: Syrphidae) in southeastern Europe, with descriptions of two new species. The Canadian Entomologist, 150, 440-464. https://doi.org/10.4039/tce.2018.21.

Nixon, K. C. (2008). ASADO, version 1.85 TNT-MrBayes Slaver version 2; mxram 200 (vl 5.30). Made available through the author (previously named WinClada, version 1.00 .08 (2002). https:// www.diversityoflife.org/winclada/.

Ricarte, A., \& Marcos-García, M. A. (2008). Los sírfidos (Diptera: Syrphidae) del Parque Nacional de Cabañeros (España): una herramienta para la gestión. Boletín de la Asociación Española de Entomología, 32(1-2), 19-32.

Ricarte, A., Nedeljković, Z., Rotheray, G. E., Lyszkowski, R. M., Hancock, E. G., Watt, K., Hewitt, S. M., Horsfield, D., \& Wilkinson, G. (2012). Syrphidae (Diptera) from the Greek island of Lesvos, with description of two new species. Zootaxa, 3175, 123. 
Ricarte, A., \& Marcos García, M. A. (2017). A checklist of the Syrphidae (Diptera) of Spain, Andorra and Gibraltar. Zootaxa, 4216, 401-440. https://doi.org/10.11646/zootaxa.4216.5.1.

Rodríguez, F., Oliver, J. L., Marín, A., \& Medina, J. R. (1990). The general stochastic model of nucleotide substitution. Journal of Theoretical Biology, 142(4), 485-501.

Rotheray, G. E. (1993). Colour guide to hoverfly larvae (Diptera, Syrphidae) in Britain and Europe. Dipterists Digest, 9, 1-156.

Sarıbıyı, S. (2014). Check list of Turkish Flower Flies (Diptera: Syrphidae). Munis Entomology \& Zoology, 9, 570-585.

Schiner, I.R. (1860). Vorläufiger Commentar zum dipterologischen Theile der Fauna Austriaca, mit einer naheren Begrundung der in derselben aufgenommenen neuen Dipteren-Gattungen. II. Wiener Entomologische Monatschrift, 4, 208-216.

Schiner, I.R. (1861). Fauna Austriaca: die Fliegen (Diptera) Nach der analytischen Methode bearb., mit der Characteristik almmilicher europäischer Gattungen, der Beechraibung aller in Deutschland vorkommenden Arten und der Aufzahlung aller bisher beschriebenen europaischen Arten. Vienna: C. Gerolds Sohn, Vienna, Austria.

Speight, M.C.D. (2018). Species accounts of European Syrphidae, 2018. Syrph the Net, the database of European Syrphidae (Diptera), vol. 10. Dublin, Syrph the Net publications.

Speight, M. C. D., Castella, E., \& Sarthou, J. P. (2016). StN 2016. In M. C. D. Speight, E. Castella, J. P. Sarthou, \& C. Vanappelghem (Eds.), Syrph the Net on CD, Issue 11. Dublin: Syrph the Net Publications.

Speight, M. C. D., \& Sarthou, J. P. (2017). StN keys for the identification of the European species of various genera of Syrphidae 2017/Clés StN pour la détermination des espèces Européennes de plusieurs genres des Syrphidae 2017. Syrph the Net, the database of European Syrphidae (Diptera), Vol. 99. Dublin, Syrph the Net publications.

Stamatakis, A. (2014). RAxML version 8: a tool for phylogenetic analysis and post-analysis of large phylogenies. Bioinformatics, 30(9): $1312-1313$

Thompson, F. C. (1999). A key to the genera of the flower flies (Diptera: Syrphidae) of the Neotropical Region including redescriptions of new genera and species and a glossary of taxonomic terms. Contributions on Entomology, International, 3, 320-378.

Thompson, F. C., \& Rotheray, G. (1998). Family Syrphidae. In L. Papp \& B. Darvas (Eds.), Contributions to a Manual of Palaearctic Diptera, Vol. 3, (pp. 81-139) Budapest.

Toksöz, A., \& Ustaoğlu, M. R. (2005). Gölcük Gölü'nün (Bozdağ, Ödemiş) Profundal Makrobentik Faunası Üzerine Araștırmalar [An investigation on the profundal macrobenthic fauna of Gölcük Lake (Bozdağ, Ödemiş)]. E.U. Journal of Fisheries \& Aquatic Sciences, 22(1-2), 173-175.
Van Eck, A. (2011). A Checklist of the hoverflies of Portugal. Boletin de la Sociedad Entomológica Aragonesa, 49, 127-144.

Van Steenis, J., \& Lucas, J. A. W. (2011). Revision of the WestPalaearctic species of Pipizella Rondani, 1856 (Diptera, Syrphidae). Dipterists Digest, 18, 127-180.

Van Steenis, J., van Zuijen, M. P., Ricarte, A., Marcos-García, M. A., Doczkal, D., Ssymank, A., \& Mengual, X. (2020). First records of Chrysotoxum volaticum Séguy, 1961 from Europe and Platycheirus marokkanus Kassebeer, 1998 from Spain (Diptera: Syrphidae) together with additional records of Spanish Chrysotoxum Meigen, 1803. Bonn zoological Bulletin, 69(1), 141-155.

Van Veen, M. P. (2004). Hoverflies of Northwest Europe: Identification keys to the Syrphidae. Utrecht: KNNV Publishing, Utrecht.

Vujić, A., Šimić, S., \& Radenković, S. (1999). Mediterranean species related to Paragus hermonensis Kaplan, 1981, with the description of Paragus gorgus spec. nov. (Diptera, Syrphidae). Volucella, 4, $20-44$.

Vujić, A., Šimić, S., \& Radenković, S. (2001). Endangered species of hoverflies (Diptera: Syrphidae) on the Balkan Peninsula. Acta entomologica serbica, 5, 93-105.

Vujić, A., Pérez-Bañón, C., Radenković, S., Ståhls, G., Rojo, S., Petanidou, T., \& Šimić, S. (2007). Two new species of the genus Merodon Meigen 1803 (Diptera: Syrphidae) from the island of Lesvos (Greece), in the eastern Mediterranean. Annales de la Société entomologique de France. (n.s.), 43, 319-326.

Vujić, A., Marcos-García, M. A., Sarıbıyık, S., \& Ricarte, A. (2011). New data on the Merodon Meigen, 1803 fauna (Diptera: Syrphidae) of Turkey, including description of a new species and changes in the nomenclatural status of several taxa. Annales de la Société entomologique de France (n.s.), 47, 78-88.

Vujić, A., Nedeljković, Z., Hayat, R., Demirözer, O., Mengual, X., \& Kazerani, F. (2017). New data on the genus Chrysotoxum Meigen (Diptera: Syrphidae) from North-East Turkey, Armenia, Azerbaijan and Iran including descriptions of three new species. Zoology in the Middle East, 63, 250-268.

Walker, F. (1852). Diptera. Part III: In W. W. Saunders (Ed.), Insecta Saundersiana: or characters of undescribed insects in the collection of William Wilson Saunders, Esq., F.R.S., F.L.S., \& c. Vol. 1 (pp. 157-252, pls. 5-6). Van Voorst, London.

Zetterstedt, J.W. (1819). Några nya Svenska Insekt-Arter fundne och beskrifne. Kungl. Svenska vetenskapsakademiens handlingar, 1, 69-86.

Publisher's note Springer Nature remains neutral with regard to jurisdictional claims in published maps and institutional affiliations. 